\title{
L-Arginine Normalizes Endothelial Function in Cerebral Vessels from Hypercholesterolemic Rabbits
}

\author{
Eugene Rossitch, Jr.," Eben Alexander III," Peter McL. Black, " and John P. Cooke* \\ ${ }^{*}$ Division of Neurosurgery, Brigham and Women's Hospital, Harvard Medical School; and ${ }^{\ddagger}$ Division of Cardiovascular Medicine, \\ Stanford University School of Medicine, Stanford, California 94305
}

\begin{abstract}
We hypothesized that normal vascular reactivity could be restored in vessels from hypercholesterolemic animals by exposing them to L-arginine, the precursor of endothelium-derived relaxing factor (EDRF). Basilar arteries were harvested from New Zealand white rabbits fed normal chow or that supplemented with $2 \%$ cholesterol for 10 wk. Vessels were cannulated for perfusion at physiologic pressure. Changes in vessel diameter were monitored by videomicroscopy.

In comparison to normal vessels, those from hypercholesterolemic animals vasoconstricted more to $\mathrm{KCl}$, endothelin $(\mathrm{E})$, and 5-hydroxytryptamine (5-HT). Conversely, vasodilation to acetylcholine (ACh) (but not that to verapamil) was significantly impaired in the hypercholesterolemic animals. In vitro administration of $L$-arginine $(3 \mathrm{mM})$ for $45 \mathrm{~min}$ normalized vasodilation to $\mathrm{ACh}$ and vasoconstriction to $\mathrm{E}, 5-\mathrm{HT}$, and $\mathrm{KCl}$ in the isolated vessels from hypercholesterolemic animals. This effect was stereospecific, since D-arginine had no effect.

To conclude, these data confirm that hypercholesterolemia attenuates endothelium-derived relaxation, and enhances the sensitivity of these vessels to vasoconstrictors. In vitro administration of $L$-arginine normalized vascular reactivity of isolated vessels from hypercholesterolemic animals. Thus, hypercholesterolemia induces a reversible endothelial dysfunction that may be corrected by supplying the precursor of EDRF, L-arginine. (J. Clin. Invest. 1991. 87:1295-1299.) Key words: L-arginine • endothelium • cholesterol • endothelium-derived relaxing factor - basilar artery
\end{abstract}

\section{Introduction}

Hypercholesterolemia induces alterations in vascular reactivity that may be manifested as an enhanced sensitivity to agonists of vasoconstriction. Vessels from hypercholesterolemic swine, monkeys, and rabbits are hyperresponsive to vasoconstrictor agents (1-6). It is thought that hypercholesterolemia potentiates vasoconstriction by inducing an endothelial impairment.

Address correspondence and reprint requests to Dr. John P. Cooke, Division of Cardiovascular Medicine, Falk Cardiovascular Research Center, Stanford University School of Medicine, 300 Pasteur Drive, Stanford, CA 94305.

Received for publication 2 April 1990 and in revised form 26 September 1990.

J. Clin. Invest.

(c) The American Society for Clinical Investigation, Inc.

$0021-9738 / 91 / 04 / 1295 / 05 \quad \$ 2.00$

Volume 87, April 1991, 1295-1299
Normally, the endothelium exerts a braking influence during stimulation of the vessel by vasoconstrictor agonists, in part, by releasing endothelium-derived relaxing factor $(E D R F)^{1}(7-10)$. However, release of endothelium-derived relaxing factor is reduced in vessels from hypercholesterolemic animals (3). For this reason, endothelium-dependent relaxation is attenuated, and vasoconstrictor responses are enhanced in vessels from hypercholesterolemic animals (1-6).

Current evidence suggests that EDRF is a nitrovasodilator, possibly nitric oxide, derived from the metabolism of L-arginine (11-14). We hypothesized that the endothelial impairment and aberrant vascular reactivity in hypercholesterolemic animals could be acutely normalized by administration of the precursor of EDRF, L-arginine. To test this hypothesis, the following study was performed.

\section{Methods}

\section{Animals}

41 New Zealand white rabbits, weighing 2.1 to $2.7 \mathrm{~kg}$, were studied. Animals were housed individually and water was provided ad libitum. Rabbits were then separated into two treatment groups: 24 were fed normal rabbit chow and 15 were fed chow containing $2 \%$ cholesterol for $10 \mathrm{wk}$ (Farmers Exchange, Framingham, MA). These protocols were approved by the Harvard Standing Committee on Animals and were performed in accordance with the recommendations of the American Association for the Accreditation of Laboratory Animal Care.

On the day of the experiment, the rabbit was given pentobarbital sodium $(30 \mathrm{mg} / \mathrm{kg}$ ) via the marginal ear vein. Heparin (200 IU) was administered with the pentobarbital. The basilar artery was then immediately removed using microinstruments under magnification.

\section{Arteriograph}

Isolated basilar arteries were mounted in an arteriograph (Living Systems Instrumentation, Burlington, VT) filled with oxygenated physiologic saline solution (PSS) at $37^{\circ} \mathrm{C}$. The proximal end of the vessel was cannulated by glass capillary tubing for perfusion with PSS delivered by a pressure-servo system to maintain luminal pressure at $60 \pm 2 \mathrm{mmHg}$. The distal end was clamped allowing luminal perfusion through the basilar branches. Changes in vessel diameter were monitored by a video system consisting of a Leitz inverted microscope (E. Leitz Inc., Rockleigh, NJ), RCA (Lancaster, PA) video camera, and a video monitor. The images were recorded and stored on VHS tape. Midplane transverse diameters were measured with a caliper directly applied to the video screen. Vessel diameter was measured distal to the proximal cannula, before the first perforating branch of the basilar artery segment. Absolute calibration was obtained using a micrometer grid placed at the level of the vessel. With this system, measurements of vessel diame-

1. Abbreviations used in this paper: EDRF, endothelium-derived relaxing factor; PSS, physiologic saline solution. 
ter are accurate to within $0.5 \mu \mathrm{m}$ (15). All drugs were added to the external bath except for acetylcholine, which was administered intraluminally by the pressure-servo syringe through the cannula. L-arginine was administered concurrently both intra- and extraluminally.

\section{Experimental protocols}

1. Vascular reactivity: effects of hypercholesterolemia. After an equilibration period (30 $\mathrm{min}$ ) in the arteriograph chamber, the vessels were perfused with PSS (at an intraluminal pressure of $60 \mathrm{mmHg}$ ) and the baseline diameter measured. Vessels were exposed to increasing concentrations of 5-hydroxytryptamine $\left(10^{-8}-10^{-5} \mathrm{M}\right)$. After the vasoconstriction stabilized, increasing concentrations of acetylcholine $\left(10^{-10}\right.$ $10^{-5} \mathrm{M}$ ) were added to the perfusate.

Subsequently, the vessels were rinsed with fresh PSS over a 45-min period. Vessels were then exposed to endothelin $\left(10^{-13}-10^{-7} \mathrm{M}\right)$ or potassium chloride $(20-80 \mathrm{mM})$. When the response stabilized to the maximum concentration of vasoconstrictor, the vessels were relaxed by increasing concentrations of verapamil $\left(10^{-8}-10^{-4} \mathrm{M}\right)$.

2. Vascular reactivity: effects of L-arginine. The above studies confirmed that endothelium-dependent vasodilation was impaired and vasoconstriction potentiated in vessels from hypercholesterolemic animals. To determine if supraphysiological concentrations of $\mathrm{L}$-arginine could normalize vascular reactivity, the following studies were performed. Vessels from hypercholesterolemic animals were exposed to L-arginine ( $3 \mathrm{mM}$ ) for $45 \mathrm{~min}$. Subsequently, vessels were exposed to 5-hydroxytryptamine, acetylcholine, endothelin, and verapamil, as outlined above.

3. Mechanism of L-arginine effect. The above studies revealed that $\mathrm{L}$-arginine potentiated endothelium-dependent vasodilation and normalized vasoconstrictor responses in vessels from hypercholesterolemic animals. To determine if the mechanism of this effect was stereospecific, the protocol 2 was repeated using D-arginine rather than L-arginine. To determine if $\mathrm{L}$-arginine affected basal vessel tone or active tension (induced by 5-hydroxytryptamine), measurements of vessel diameter were made before and during the administration of $L$-arginine To determine if $\mathrm{L}$-arginine augmented endothelial response in normal vessels, tissues were contracted by 5-hydroxytryptamine and relaxed with acetylcholine before and after exposure to L-arginine. To determine if $\mathrm{L}$-arginine had a direct effect on vascular smooth muscle responsiveness, in another series of experiments, vessels from normal animals were denuded of endothelium. The endothelium was removed by perfusing the vessels for $1 \mathrm{~min}$ with distilled water $(1 \mathrm{ml} / \mathrm{min})$. Vessels were then contracted by 5-hydroxytryptamine or potassium chloride and relaxed with verapamil before and after exposure to $\mathrm{L}$-arginine.

\section{Drugs}

The following drugs were used: 5-hydroxytryptamine creatinine sulfate, acetylcholine chloride, verapamil, D-arginine hydrochloride, L-arginine hydrochloride (Sigma Chemical Co., St. Louis, MO), endothelin (Peninsula Laboratories, Belmont, CA), and pentobarbitol sodium (Abbott Laboratories, Chicago, IL). All drugs were soluble in distilled water or PSS. Stock solutions of hygroscopic acetylcholine chloride were prepared and stored frozen as previously described (16). All other agents were prepared on the day of the experiment and stored on ice until use. Drugs were added to the arteriograph chamber or pressureservo syringe in volumes less than $75 \mu 1$. Concentrations are expressed as the final molar concentration in the bath solution or perfusate.

\section{Data analysis}

Data are expressed as mean \pm SE. Each dose-response curve was characterized by determining the $\mathrm{EC}_{50}$, i.e., the dose of the drug inducing a half-maximal vasoconstriction (or vasodilation), and by determining the maximal response to the drug. Multiple comparisons were avoided by comparing only the $\mathrm{EC}_{50}$ and maximal responses. The data were analyzed using Student's $t$ tests; for multiple group comparisons an analysis of variance was performed followed by a Newman-Keuls test.

\section{Results}

\section{Experimental protocols}

1. Vascular reactivity: effects of hypercholesterolemia. The initial vessel diameter was not different between the control and cholesterol groups: $764 \pm 8.4 \mu \mathrm{m}, n=12$; vs. $821 \pm 10.4 \mu \mathrm{m}, n$ $=6$, respectively.

Endothelium-dependent vasodilation was attenuated in vessels from hypercholesterolemic animals. The sensitivity to acetylcholine was reduced with a 150 -fold rightward shift in the dose-response relationship (Table I, Fig. 1 a). The maximal response to acetylcholine was unaffected. The impairment in endothelial-mediated vasodilation was not due to a reduction in the ability of the vascular smooth muscle to relax. Indeed, vasodilation to verapamil (endothelium-independent) tended to be greater in the vessels from hypercholesterolemic animals (Table I, Fig. $1 b$ ).

Vasoconstrictor responses were enhanced in vessels from hypercholesterolemic animals. Maximum vasoconstriction to 5-hydroxytryptamine was increased by $74 \%$, although the sensitivity $\left(\mathrm{EC}_{50}\right)$ was unaffected (Table II, Fig. $2 a$ ). The sensitivity to endothelin was increased, as manifested by a 100 -fold leftward shift in the dose response relationship, but the maximum vasoconstriction was not altered (Table II, Fig. 2 b). Similarly, the sensitivity to potassium chloride was increased, but the maximum vasoconstriction was unchanged (Table II, Fig. $2 c$ ).

2. Vascular reactivity: effects of $\mathrm{L}$-arginine. The baseline diameter for the arginine treated vessels was $845 \pm 7.6 \mu \mathrm{m}, n$ $=6$, which was not different from the other two experimental groups.

Endothelium-dependent vasodilation was normalized in vessels from hypercholesterolemic animals exposed to L-arginine (Table I). Vessels from hypercholesterolemic animals exposed to L-arginine were more sensitive to acetylcholine than those exposed to vehicle, with a leftward shift in the $\mathrm{EC}_{50}$ value of more than two orders of magnitude (Table I, Fig. 3).

Vasoconstrictor responses were also normalized in vessels from hypercholesterolemic animals exposed to L-arginine. Maximum vasoconstriction to 5-hydroxytryptamine was not

Table I. Endothelium-dependent Vasodilation Is Attenuated by Hypercholesterolemia and Restored by L-Arginine

\begin{tabular}{|c|c|c|c|c|c|c|}
\hline \multirow[b]{2}{*}{ Condition* } & \multicolumn{3}{|c|}{ Acetylcholine } & \multicolumn{3}{|c|}{ Verapamil } \\
\hline & $n$ & $\mathrm{EC}_{30}{ }^{*}$ & $\operatorname{Max}^{5}$ & $n$ & $\mathrm{EC}_{50}{ }^{*}$ & $\operatorname{Max}^{5}$ \\
\hline Control & 12 & $9.1 \pm 0.9$ & $126 \% \pm 68$ & 5 & $5.6 \pm 0.7$ & $103 \% \pm 26$ \\
\hline Chol & 6 & $6.9 \pm 1.0^{\prime \prime}$ & $86 \% \pm 19$ & 5 & $6.1 \pm 1.0$ & $157 \% \pm 36$ \\
\hline Arg & 5 & $9.7 \pm 0.7^{1}$ & $103 \% \pm 32$ & 5 & $5.7 \pm 0.3$ & $136 \% \pm 28$ \\
\hline
\end{tabular}

* Control, vessels harvested from normal animals; Chol, vessels harvested from hypercholesterolemic animals and exposed to vehicle; Arg, vessels harvested from hypercholesterolemic animals and exposed to L-arginine $\left(3 \times 10^{-3} \mathrm{M}\right)$ in vitro. ${ }^{\ddagger} E C_{50}$ for acetylcholine and verapamil is expressed as dose $(-\log M)$ inducing the half-maximal vasodilation. $\mathrm{Max}$, maximum vasodilation, expressed as a percentage of relaxation of the vasoconstriction induced by 5-hydroxytryptamine (acetylcholine data) or endothelin (verapamil data). "Significantly different from control value; $P<0.01$. 'Significantly different from Chol group; $P<0.01$. 

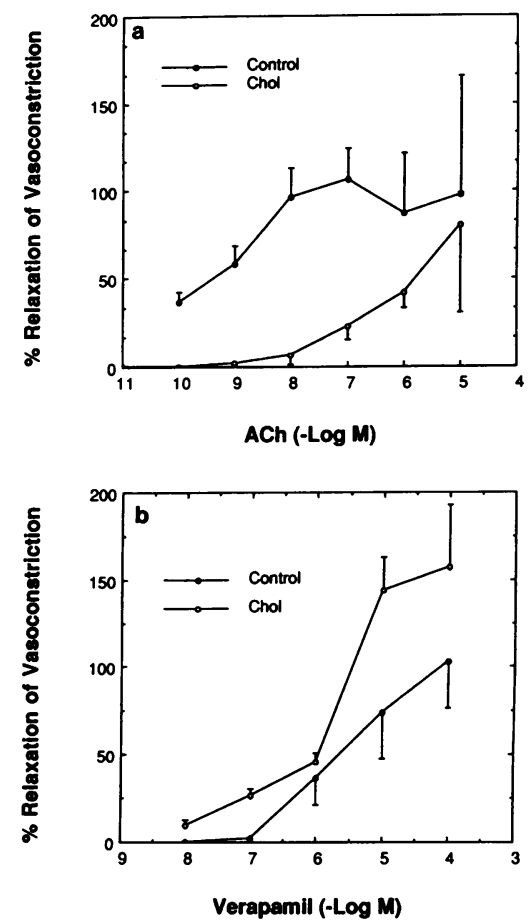

laxation of the vasoconstriction induced by endothelin. Neither the maximum vasodilation nor the $\mathrm{EC}_{50}$ value were significantly different between the two groups ( $n=5$ in each group).

different from the normal vessels (Table II). The sensitivity to endothelin was also normalized (Table II, Fig. 4).

3. Mechanism of $\mathrm{L}$-arginine effects. $\mathrm{L}$-arginine did not induce vasodilation directly. Vasoconstriction to 5-hydroxytryptamine $\left(10^{-5} \mathrm{M}\right)$ was not relaxed when the vessels were perfused with saline solution containing L-arginine $3 \times 10^{-3} \mathrm{M}$ (vessel diameter of $718 \pm 79$ vs. $712 \pm 79 \mu \mathrm{m}$ after perfusion with vehicle control vs. L-arginine; $n=2, P=\mathrm{NS}$ ). In vessels denuded of endothelium, contractions to potassium chloride ( $n$ $=3)$ and those to 5-hydroxytryptamine $(n=3)$ and vasodilation to verapamil $(n=6)$ were not different after exposure to L-arginine (Table III).

The effect of $\mathrm{L}$-arginine was stereospecific. In vessels from hypercholesterolemic animals, D-arginine $\left(3 \times 10^{-3} \mathrm{M}\right)$ did not alter basal diameter, nor did it affect vasoconstriction to 5-hydroxytryptamine or vasodilation to acetylcholine $(n=2$; data not shown). The effect of L-arginine was confined to vessels from hypercholesterolemic animals. L-arginine $\left(3 \times 10^{-3} \mathrm{M}\right)$ did not affect vasodilation to acetylcholine in normal tissues $(n$ $=2$; data not shown).

\section{Discussion}

Hypercholesterolemia alters vascular reactivity. In isolated vessels and in vivo, responsiveness to vasoconstrictor agonists is enhanced and endothelium-dependent relaxations are attenuated (1-6). We hypothesized that these abnormalities could be acutely reversed by administering the precursor of EDRF, $\mathrm{L}$-arginine. We tested this hypothesis in this investigation and found that: $(a)$ hypercholesterolemia impairs endothelium-dependent vasorelaxation and enhances vasoconstrictor response; and $(b)$ the in vitro administration of $\mathrm{L}$-arginine potentiates endothelium-dependent relaxation in isolated vessels from hypercholesterolemic animals and acutely normalizes vasoconstrictor response. This effect of $L$-arginine is stereospecific, since D-arginine did not affect vascular reactivity. The effect of $L$-arginine was not due to a direct action on the vascular smooth muscle, since it had no effect on vasoconstriction or vasodilation in the absence of endothelium. Furthermore, L-arginine had no effect on relaxation to verapamil, the action of which is independent of the endothelium.

L-arginine had no effect on reactivity of vessels from normal animals. This finding is consistent with other reports that L-arginine has little or no effect on vascular reactivity of normal tissue (17-20). These observations would suggest that under most circumstances, there is sufficient endothelial stores of L-arginine to saturate the nitric oxide forming enzyme.

However, under certain circumstances, the administration of $\mathrm{L}$-arginine is critical for restoration of endothelium-dependent relaxation. Endothelial cells cultured in L-arginine-deficient medium release little nitric oxide until the cells are exposed to L-arginine (12). Antagonists of arginine metabolism attenuate endothelium-dependent vasodilation, reduce the release of nitric oxide from isolated vessels, and increase vascular resistance in vivo; these abnormalities are reversed by administration of L-arginine (20). L-arginine restores responsiveness in vascular rings made refractory to endothelial stimulation by prolonged exposure to calcium ionophore (21).

We now report that L-arginine acutely normalizes endothelium-dependent relaxation in isolated vessels from hypercholesterolemic animals. This finding is consistent with our

Table II. Vasoconstrictor Responses Are Enhanced by Hypercholesterolemia and Normalized by L-Arginine

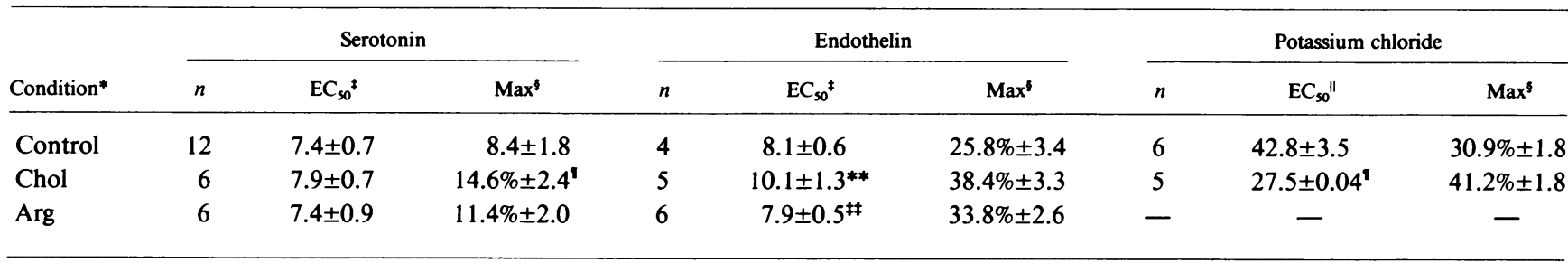

* Control, vessels harvested from normal animals; Chol, vessels harvested from hypercholesterolemic animals and exposed to vehicle; $\mathrm{Arg}$, vessels harvested from hypercholesterolemic animals and exposed to L-arginine $\left(3 \times 10^{-3} \mathrm{M}\right)$ in vitro. ${ }^{\ddagger} E C_{50}$ for 5 -HT and ET is expressed as the dose $(-\log \mathrm{M})$ inducing the half-maximal vasoconstriction. ${ }^{\S} \mathrm{Max}$, maximum vasoconstriction, expressed as a percentage of initial vessel diameter. " $E C_{50}$ for $\mathrm{KCl}$ is expressed as dose $(\mathrm{mM})$ inducing the half-maximal vasoconstriction. ' Significantly different from control value, $P<0.05$.

** Significantly different from control value, $P<0.01$. ${ }^{\sharp}$ Significantly different from $C h o l$ group, $P<0.01$. 

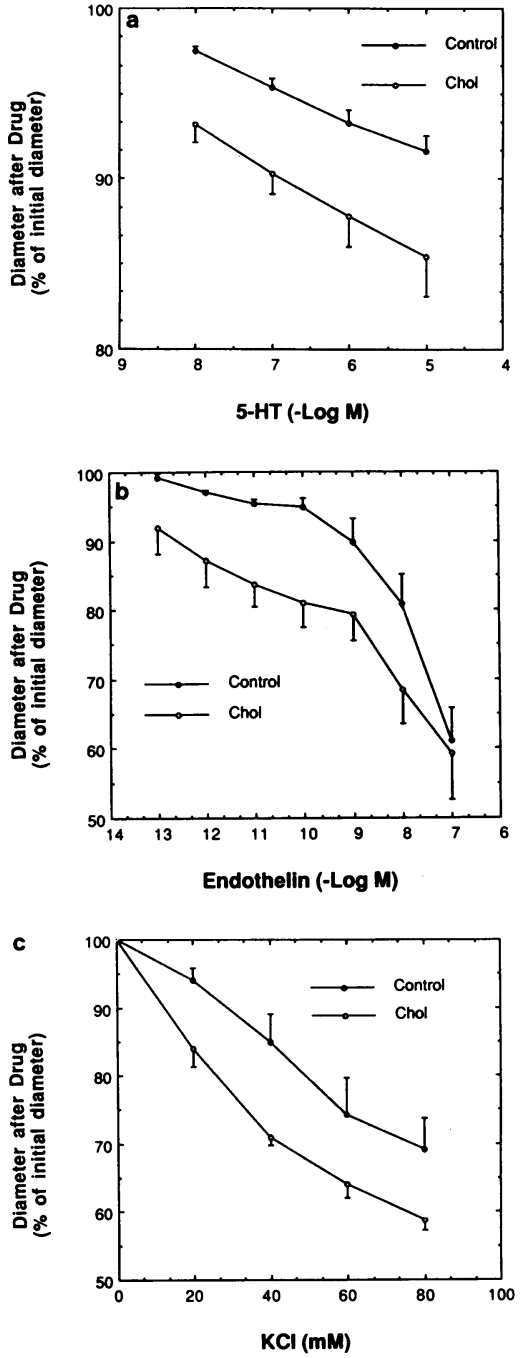

Figure 2. (a) Vasoconstriction to serotonin (5-hydroxytryptamine [5-HT]) is enhanced in the basilar artery of the hypercholesterolemic rabbit. Diameter after exposure to the vasoconstrictor is expressed as a percentage $( \pm$ SEM) of the initial diameter. The maximum vasoconstriction to serotonin was greater in vessels from hypercholesterolemic animals $(14.6 \pm 2.4, n=6$ in the cholesterol group vs. $8.4 \pm 1.8, n=12$ in the control group; $P$ $<0.05$ ). (b) Vasoconstriction to endothelin is enhanced in the basilar artery of the hypercholesterolemic rabbit. Diameter after exposure to the vasoconstrictor is expressed as a percentage $( \pm$ SEM $)$ of the initial diameter. The vessels from hypercholesterolemic animals exhibited a greater sensitivity to endothelin $\left(\mathrm{EC}_{50}\right.$ of $10.1 \pm 1.3, n=5$ in the hypercholesterolemic group vs.

$8.1 \pm 0.6, n=4$ in the control group; $P$ $<0.01)$. (c) Vasoconstriction to potassium chloride $(\mathrm{KCl})$ is enhanced in the basilar artery of the hypercholesterolemic rabbit. Diameter after exposure to the vasoconstrictor is expressed as a percentage $( \pm$ SEM) of the initial diameter. The vessels from hypercholesterolemic animals exhibited a greater sensitivity to $\mathrm{KCl}\left(\mathrm{EC}_{50}\right.$ of $27.5 \pm 0.04, n=5$ in the hypercholesterolemic group vs. $42.8 \pm 3.5, n=6$ in the control group; $P<0.05$ )

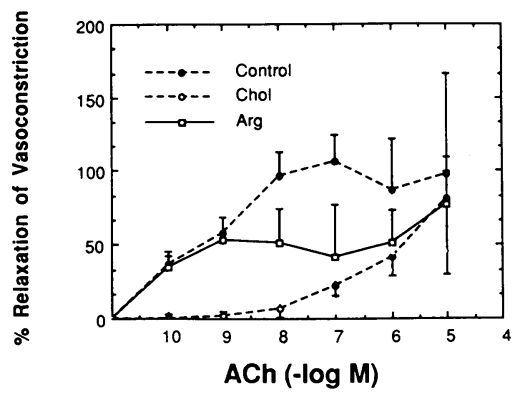

Figure 3. L-arginine potentiates the response to acetylcholine in vessels from hypercholesterolemic animals. Data from Fig. 1 a (dashed lines) are superimposed upon the dose response to acetylcholine of $\mathrm{L}$-arginine-treated vessels from hypercholesterolemic animals. Vasodilation by acetylcholine $(A C h)$ is expressed as a percentage ( \pm SEM) relaxation of the vasoconstriction induced by 5-hydroxytryptamine. The sensitivity to $\mathrm{ACh}$ was not different in the arginine-treated vessels of hypercholesterolemic animals in comparison to normal vessels (Table I). By contrast, the sensitivity to ACh was significantly less in the vehicle-treated vessels from hypercholesterolemic animals in comparison to either of the other two groups (Table I).

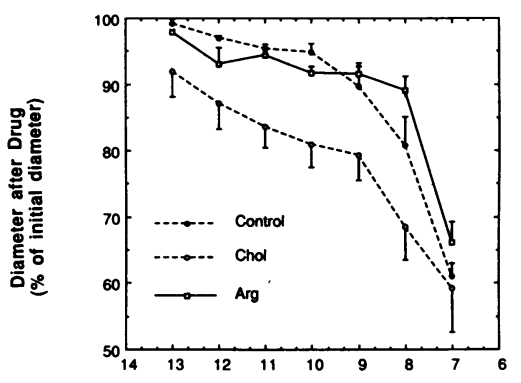

Figure 4. L-arginine normalizes the response to endothelin in vessels from hypercholesterolemic animals. Data from Fig. $2 b$ (dashed lines) are superimposed upon the dose response to endothelin of L-arginine-treated vessels from hypercholesterolemic animals $(n=6)$. Vasoconstriction is ex-

pressed as a percentage $( \pm$ SEM) of the initial diameter. The sensitivity to endothelin was not different in the arginine-treated vessels of hypercholesterolemic animals in comparison to normal vessels (Table II). By contrast, the sensitivity to endothelin was significantly greater in vehicle-treated vessels from hypercholesterolemic animals in comparison to either of the other groups (Table II).

previous observation that intravenous administration of $\mathrm{L}$-arginine augments hindlimb blood flow in response to acetylcholine in hypercholesterolemic rabbits (22). The data suggest that exposure to elevated levels of cholesterol reversibly interferes with the synthesis and/or release of EDRF, just as it interferes with other synthetic functions of the endothelium (23).

We also found that administration of $\mathrm{L}$-arginine normalized responsiveness to vasoconstrictor agonists. Normally, the endothelium modulates vasoconstriction to aggregating platelets, 5-hydroxytryptamine, norepinephrine, endothelin, and other vasoconstrictors, in part, by releasing EDRF (7-10). Both endothelin and 5-hydroxytryptamine are known to release EDRF as well as to induce contraction of the vascular smooth muscle $(7,8)$. Therefore, in normal vessels, the vasoconstriction to endothelin or to 5-hydroxytryptamine is attenuated by the simultaneous release of EDRF. Since the release of EDRF is reduced in vessels from hypercholesterolemic animals, this likely explains the heightened response to endothelin and to serotonin. These findings are consistent with the work of other investigators documenting the increased sensitivity to vasoconstrictors in hypercholesterolemic states $(1,2,4,5)$. We found that administration of L-arginine to vessels from hypercholesterolemic animals normalized vasoconstriction to endothelin and to serotonin. Since L-arginine augmented the release of EDRF by acetylcholine, it is likely that the release of EDRF by endothelin or 5-hydroxytryptamine is also augmented. This would explain the reversal by L-arginine of the heightened vasoconstrictor response of hypercholesterolemic vessels.

Since the vasoconstrictors were delivered by perfusate flowing through the vessel, it is quite likely that the response to these agonists was influenced by the effects of flow on the endothelium. Flow is known to induce vasodilation, which appears to be largely due to the release of $\operatorname{EDRF}(24,25)$. Therefore, in our system, the release of EDRF by flow should attenuate vasoconstriction and augment vasodilation. It is not known whether flow-induced release of EDRF is reduced in hypercholesterolemia, but in the presence of overt atherosclerosis, flowmediated vasodilation is reduced in human coronary arteries (26-28). Therefore, the abnormal vascular reactivity we observed in the vessels from hypercholesterolemic rabbits could be due to abnormalities in flow-mediated vasodilation. Nevertheless, this interpretation is consistent with our conclusions 
Table III. L-Arginine Has no Effect Upon Vascular Reactivity in Vessels Denuded of Endothelium

\begin{tabular}{|c|c|c|c|c|c|c|}
\hline \multirow[b]{3}{*}{ Condition* } & \multicolumn{4}{|c|}{ Vasoconstriction } & \multirow{2}{*}{\multicolumn{2}{|c|}{$\begin{array}{c}\text { Vasodilation } \\
\text { Verapamil }\end{array}$}} \\
\hline & \multicolumn{2}{|c|}{ 5-hydroxytryptamine } & \multicolumn{2}{|c|}{ Potassium chloride } & & \\
\hline & $\mathrm{EC}_{\text {so }}{ }^{*}$ & $\operatorname{Max}$ & $\mathrm{EC}_{\mathbf{s 0}}{ }^{*}$ & $\operatorname{Max}$ & $\mathrm{EC}_{\text {so }}{ }^{\prime \prime}$ & $\operatorname{Max}$ \\
\hline Control $(n=3)$ & $7.2 \pm 0.3$ & $10.8 \pm 1.1$ & $32 \pm 2.2$ & $28.5 \pm 6.6$ & $7.3 \pm 0.1$ & $139 \pm 6.9$ \\
\hline $\operatorname{Arg}(n=3)$ & $7.3 \pm 0.3$ & $8.9 \pm 0.7$ & $43 \pm 9.8$ & $30.2 \pm 7.0$ & $7.1 \pm 0.2$ & $153 \pm 12.2$ \\
\hline
\end{tabular}

* Control, vessels exposed to vehicle; $A r g$, vessels exposed to L-arginine $3 \times 10^{-3} \mathrm{M}$ in vitro. ${ }^{\ddagger} E C_{50}$ for 5 -HT is expressed as the dose (-log M) inducing the half-maximal vasoconstriction. $E C_{50}$ for $\mathrm{KCl}$ is expressed as dose (mM) inducing the half-maximal vasoconstriction. ${ }^{8} \mathrm{Max}(5-\mathrm{HT}$, $\mathrm{KCl}$ ), maximum vasoconstriction, expressed as a percentage of initial vessel diameter. Max (verapamil), maximum vasodilation, expressed as a percentage relaxation of the vasoconstriction induced by $\mathrm{KCl}$. " $E C_{50}$ for verapamil is expressed as dose $(-\log \mathrm{M})$ inducing the half-maximal vasodilation.

that the abnormal vascular reactivity we observed is due to an endothelial dysfunction, which is corrected by exposure to $\mathrm{L}$ arginine.

In summary, we find that endothelium-dependent vasodilation is impaired and vasoconstriction is enhanced in the basilar artery of hypercholesterolemic rabbits. These abnormalities are acutely reversed by the administration of $L$-arginine, the precursor of EDRF. The data suggest that the abnormal vascular responses in hypercholesterolemic animals are due to a reversible reduction in intracellular arginine availability or metabolism.

\section{References}

1. Henry, P. D., and M. Yokoyama. 1980. Supersensitivity of atherosclerotic rabbit aorta to ergonovine. Mediation by a serotonergic mechanism. J. Clin. Invest. 66:306-313.

2. Shimokawa, H., H. Tomoike, S. Nabeyama, H. Yamamoto, H. Araki, M. Nakamura, Y. Ishii, and K. Tanaka. 1983. Coronary artery spasm induced in atherosclerotic miniature swine. Science (Wash. DC). 221:560-562.

3. Verbeuren, T. H., F. H. Jordaens, L. L. Zonnekeyn, G. E. VanHove, M. C. Coene, and A. G. Herman. 1986. Effect of hypercholesterolemia on vascular reactivity in the rabbit. Circ. Res. 58:552-564.

4. Yamamoto, Y., H. Tomoike, K. Egashira, and M. Nakamura. 1987. Attenuation of endothelium-related relaxation and enhanced responsiveness of vascular smooth muscle to histamine in spastic coronary arterial segments from miniature pigs. Circ. Res. 61:772-778.

5. Heistad, D. D., M. L. I. Armstrong, M. L. Marcus, D. J. Piegors, and A. L Mark. 1984. Augmented responses to vasoconstrictor stimuli in hypercholesterolemic and atherosclerotic monkeys. Circ. Res. 54:711-718.

6. Shimokawa, H., P. Kim, and P. M. Vanhoutte. 1988. Endothelium-dependent relaxation to aggregating platelets in isolated basilar arteries of control and hypercholesterolemic pigs. Circ. Res. 63:604-612.

7. Warner, T. D., J. A. Mitchell, G. deNucci, and J. R. Vane. 1989. Endothelin-1 and endothelin-3 release EDRF from isolated perfused arterial vessels of the rat and rabbit. J. Cardiovasc. Pharmacol. 13(Suppl):585-588.

8. Houston, D. S., J. T. Shepherd, and P. M. Vanhoutte 1985. Adenine nucleotides, serotonin, and endothelium-dependent relaxations to platelets. Am. J. Physiol. 248:H389-H395.

9. Katusic, Z. S., J. T. Shepherd, and P. M. Vanhoutte. 1984. Vasopressin causes endothelium-dependent relaxations of the canine basilar artery. Circ. Res. 55:575-579.

10. Cocks, T. M., and J. A. Angus. 1983. Endothelium dependent relaxation of coronary arteries by noradrenaline and serotonin. Nature (Lond.). 305:627630.

11. Palmer, R. M. J., A. G. Ferridge, and S. Moncada. 1987. Nitric oxide release accounts for the biological activity of endothelium-derived relaxing factor. Nature (Lond.). 327:524-526.
12. Palmer, R. M. J., D. S. Ashton, and S. Moncada. 1988. Vascular endothelial cells synthesize nitric oxide from L-arginine. Nature (Lond.). 33:664-666.

13. Ignarro, L. H., R. E. Byrns, G. M. Buga, and K. S. Wood. 1987. Endothelium-derived relaxing factor from pulmonary artery and vein possesses pharmacologic and chemical properties identical to those of nitric oxide radical. Circ. Res. 61:866-879.

14. Furchgott, R. F. 1988. Studies on relaxation of rabbit aorta by sodium nitrite: the basis for the proposal that the acid-activatable inhibitory factor from retractor penis is inorganic nitrite and the endothelium-derived relaxing factor is nitric oxide. In Mechanisms of Vasodilation. P. M. Vanhoutte, editor. Raven Press, New York. 401-414.

15. Yamamoto, H., C. Bossaller, and J. Cartwright, Jr. 1988. Videomicroscopic demonstration of defective cholinergic arteriolar vasodilation in atherosclerotic rabbit. J. Clin. Invest. 81:1752-1758.

16. Ignarro, L. J., T. M. Burke, K. S. Wood, and P. J. Kadowitz. 1984. Association between cyclic GMP accumulation and acetylcholine elicited relaxation of bovine intrapulmonary artery. J. Pharmacol. Exp. Ther. 228:682-690.

17. Thomas, G., R. Vargas, B. Wroblewska, and P. W. Ramwell. 1989. Role of the endothelium and arginine peptides on the vasomotor response of porcine internal mammary artery. Life Sci. 44:1823-1830.

18. Aisaka, K., S. S. Gross, O. W. Griffith, and R. Levi. 1989. N ${ }^{\mathrm{G}}$-methylarginine, an inhibitor of endothelium-derived nitric oxide synthesis, is a potent pressor agent in the guinea pig: does nitric oxide regulate blood pressure in vivo? Biochem. Biophys. Res. Commun. 160:881-886.

19. Amezcua, J. L., R. M. J. Palmer, B. M. De Souza, and S. Moncada. 1989. Nitric oxide synthesized from L-arginine regulates vascular tone in the coronary circulation of the rabbit. Br. J. Pharmacol. 97:1119-1124.

20. Rees, D. D., R. M. J. Palmer, and S. Moncada. 1989. Role of endothelium-derived nitric oxide in the regulation of blood pressure. Proc. Natl. Acad. Sci. USA. 86:3375-3378.

21. Gold, M. E., K. S. Wood, G. M. Buga, R. E. Byrns, and L. J. Ignarro. 1989. L-arginine causes whereas L-argininosuccinic acid inhibits endothelium-dependent vascular smooth muscle relaxation. Biochem. Biophys. Res. Commun. 161:536-543.

22. Girerd, X. J., A. T. Hirsch, J. P. Cooke, and M. A. Creager. 1989. L-arginine augments endothelium-dependent vasodilation in cholesterol fed rabbits. Circulation. 80(Suppl II):II-280.

23. Dainiak, N., H. B. Warren, S. Kreczko, L. Feldman, J. Lawler, A. M. Cohen, and P. F. Davies. 1988. Acetylated lipoproteins impair erythroid growth factor release from endothelial cells. J. Clin. Invest. 81:834-843.

24. Pohl, V., J. Holtz, R. Busse, and E. Bassenge. 1986. Crucial role of the endothelium in the vasodilator response to increased flow in vivo. Hypertension. 8:37-44.

25. Cooke, J. P., J. S. Stamler, N. Andon, P. F. Davies, and J. Loscalzo. 1990 Flow stimulates endothelial cells to release a nitrovasodilator that is potentiated by reduced thiol. Am. J. Physiol. 28:H804-H812.

26. Cox, D. A., J. S. Vita, C. B. Treasure, D. R. Fish, R. W. Alexander, P. Ganz, and A. P. Selwyn. 1989. Atherosclerosis impairs flow-mediated dilation of coronary arteries in humans. Circulation. 80:458-465.

27. Drexler, J., A. M. Zeiher, H. Wollschlager, T. Meinertz, H. Just, and T. Bonzel. 1989. Flow-dependent coronary artery dilation in humans. Circulation. 80:466-474.

28. Nabel, E. G., A. P. Selwyn, and P. Ganz. 1990. Large coronary arteries in humans are responsive to changing blood flow: an endothelium-dependent mechanism that fails in patients with atherosclerosis. J. Am. Coll. Cardiol. 16:349-356. 